\title{
Modélisation des effets hydrochimiques à long terme des dépôts acides et des reboisements dans les bassins versants du Mont-Lozère (Sud de la France) Modelling the long-term effects of acid deposition and afforestation in the Mont-Lozere catchments (southern France)
}

\author{
P. Durand, E. Lelong et C. Neal
}

Volume 5, numéro 2, 1992

URI : https://id.erudit.org/iderudit/705130ar

DOI : https://doi.org/10.7202/705130ar

\section{Aller au sommaire du numéro}

\section{Éditeur(s)}

Université du Québec - INRS-Eau, Terre et Environnement (INRS-ETE)

ISSN

0992-7158 (imprimé)

1718-8598 (numérique)

\section{Découvrir la revue}

\section{Citer cet article}

Durand, P., Lelong, E. \& Neal, C. (1992). Modélisation des effets hydrochimiques à long terme des dépôts acides et des reboisements dans les bassins versants du Mont-Lozère (Sud de la France). Revue des sciences de l'eau / Journal of Water Science, 5(2), 229-245. https://doi.org/10.7202/705130ar

\section{Résumé de l'article}

L'évolution de la chimie des sols et des eaux dans trois petits bassins-versants subméditerranéens granitiques recevant d'importants apports atmosphériques de $\mathrm{SO}_{4}$ et de poussières sahariennes a été simulée, de 1845 à 2125, en utilisant le modèle MAGIC. Ce modèle biogéochimique global comporte une série de relations d'équilibre entre les phases gazeuse, liquide et solide (adsorption de soufre, solubilité de l'aluminium, échanges cationiques, système

$\mathrm{CO}_{2} /$ carbonates, dissociation des acides organiques) et une comptabilisation des flux d'éléments (entrées atmosphériques, sorties hydrologiques, immobilisation biologique, altération). Les bassins versants diffèrent par l'historique récente de leur utilisation par l'homme : la pelouse pâturée qui les recouvraient en 1845 n'a subsisté que dans un bassin : elle a été remplacée vers 1930 par une pessière dans l'un des bassins, et colonisée, après abandon, par une hêtraie dans l'autre. Les simulations montrent que le bassin couvert de pelouse a peu souffert de l'augmentation de l'acidité des pluies au cours du XX siècle, et pourrait subir les apports actuels pendant plus de 100 ans sans dommage. Les deux bassins forestiers s'acidifient fortement depuis 1970 par les effets combinés de la pollution et des reboisements. Pour la pessière, seule une réduction d'au moins $60 \%$ des apports soufrés permettrait d'inverser le processus. Le vieillissement naturel de la hêtraie produirait une amélioration même sans réduction des dépôts acides, en diminuant le taux d'assimilation de cations. Les deux facteurs acidifiants agissent donc en synergie, mais dans le cas de la pessière l'augmentation des apports acides due à l'effet filtrant des frondaisons est prépondérant, alors que dans la hêtraie, la forte immobilisation cationique dans la biomasse perenne jolie un plus grand rôle. 


\title{
Modélisation des effets hydrochimiques à long terme des dépôts acides et des reboisements dans les bassins versants du Mont-Lozère (Sud de la France)
}

\author{
Modelling the long-term effects \\ of acid deposition and afforestation \\ in the Mont-Lozere catchments (southern France)
}

P. DURAND1, F. LELONG2, C. NEAL1

Reçu le 20 avril 1991, accepté pour publication le 20 février $1992^{\star}$.

SUMMARY

Introduction. The combined acidifying effects of afforestation and acid deposition are well documented for sites in North-Western Europe and North America. In acid-sensitive mediterranean areas, acidification has been delayed by lower deposition rates and alkaline aeolian dust input to the ecosystems. Here, the evolution of stream water and soil chemistry, from 1845 to 2125, in three small submediterranean catchments of Southern France, is assessed using the MAGIC model.

Sites and methods. Three granitic catchments have been monitored since 1981 in the South-Eastern Massif Central. The mean elevation is $1300 \mathrm{~m}$ above sea level and the solis are rankers and acid brown earth. The catchments are submitted to heavy atmospheric deposition of industrial acidic oxides (mainly sulphate: more than $20 \mathrm{~kg}^{-h^{-}} \mathrm{T}_{\text {-year }}{ }^{-1}$ of $\mathrm{S}-\mathrm{SO}_{4}$ ) and of alkaline saharan dust. The land use was similar in all the catchments up to 1930, and consisted mainly of extensive sheep grazing on semi natural grassiand. Then one catchment (19.5 ha) was afforested with conifers (spruce), another calchment (54 ha) was abandoned and progressively settled by a beech coppice, while the other catchment (81 ha) remained covered with grassland. MAGIC is a lumped, process-oriented biogeochemical model, where the soil physical and chemical characteristics are described by a single set of mean variables. The processes in soils are modelled by a series of equilibrium equations : Aluminium solubility, cation exchange, sulphate adsorption, $\mathrm{CO}_{2}$ and carbonates equilibria, organic acids dissociation. The changes in the stocks of elements are calculated from the main input, output and internal fluxes : atmospheric deposition, river load, biological uptake and weathering. The calibration aims to get the best fit between measured and simulated values to the otarget variabies $n$, i.e., the present day

1. Institute of Hydrology, Wallingford, $0 \times 108 B 8$ (U.K.).

2. Centre des Sciences de la Terre, 21100 Dijon (France).

* Les commentaires seront reçus jusqu'au 30 décembre 1992. 
exchangeable cations amounts in soils and river chemical composition. Hindcast and forecast runs need historical and prospective scenarios for atmospheric deposition, dry deposition factor, nutrient uptake and discharge. Here, the growth of the forest was modelled by increasing dry deposition factor and nutrient uptake and decreasing the discharge according to the field observations. The sequence of sulphate deposition was derived from $\mathrm{SO}_{2}$ emission data. Three scenarios were tested in the forecasts : a constant deposition at the present level, a $30 \%$ reduction and a $60 \%$ reduction within 2010 . In both cases, the nutrient uptake of the spruce stand and grassiand was kept constan!, while those of the beech coppice was progressively decreased to simulate a natural ageing of the forest.

Results. The model successfully reproduced the chemistry of the catchments. The values of the optimised parameters suggest that:

- the soils of the area have a very high sulphate adsorption capacity ;

- the weathering rates are similar in the three catchments except for the calcium (lower in the beech catchment) and the magnesium (much higher in the spruce calhement) ;

- the initial conditions calculated by the model for $\mathbf{1 8 4 5}$ are slightly different; the alkalinity and calions concentrations are lower, and the base saturation higher, in the beech calchment.

The simulations show that for the grassland catchment, there has been little change due to increasing of $\mathrm{SO}_{4}$ wet deposition over the last 100 years. The simulations for the other two catchments (beech and spruce stands) show that they have been acidifying since 1970 due to the combined effects of air pollution and afforestation. This trend could lead to a severe decline of soil hase saturation and streamwater quality by 2050 . Concerning the coniferous catchment, only a $60 \%$ reduction of the sulphur input could allow a recovery. The natural ageing of the beech stand, resulting in a decrease of the biological uptake, would permit a recovery even under conslant sulphur deposition.

Conclusion. This exercise show that although the effects afforestation and acid deposition are synergetical and difficult to distinguish, their relative importance in acidification processes varies according to the vegetation. In the spruce catchment, the enhanced deposition due to the scavenging properties of the canopy is the determining factor. If no emission reduction occurs and no mitigating measures are taken, the decline of soil and water quality is a serious threat. In the beech calchment, the dynamics of biological uptake prevail, because the trees store a large amount of base cations. If the stand is allowed to age, a recovery can be observed even if no sulphur emission reduction occurs. This is aiso partly due, in this context, to the scavenging of the alkaline azolian dust.

Key-words : acidification, afforestation, catchment, modelling, MAGIC, granite, Mont-Lozere, hydrochemistry.

L'évolution de la chimie des sols et des eaux dans trois petits bassinsversants subméditerranéens granitiques recevant d'importants apports atmosphériques de $\mathrm{SO}_{4}$ et de poussières sahariennes a été simulée, de 1845 à 2125, en utilisant le modèle MAGiC. Ce modèle biogéochimique global comporte une série de relations d'équilibre entre les phases gazeuse, liquide et solide (adsorption de soufre, solubilité de l'aluminium, échanges cationiques, système $\mathrm{CO}_{2}$ /carbonates, dissociation des acides organiques) et une comptabilisation des flux d'éléments (entrées atmophériques, sorties hydrologiques, immobilisation biologique, altération). Les hassins versants diffèrent par l'historique récente de leur utilisation par l'homme : la pelouse pâturée qui 
les recouvraient en 1845 n'a subsisté que dans un bassin : elle a été remplacée vers 1930 par une pessière dans l'un des bassins, et colonisée, après abandon, par une hêtraie dans l'autre. Les simulations montrent que le bassin couvert de pelouse a peu souffert de l'augmentation de l'acidité des pluies au cours du XXe siècle, et pourrait subir les apports actueis pendant plus de $\mathbf{1 0 0}$ ans sans dommage. Les deux bassins forestiers s'acidifient forte ment depuis 1970 par les effets combinés de la pollution et des reboisements. Pour la pessière, seule une réduction d'au moins $60 \%$ des apports soufrés permettrait d'inverser le processus. Le vieillissement naturel de la hêtraie produirait une amélioration même sans réduction des dépôts acides, en diminuant le taux d'assimilation de cations. Les deux facteurs acidifiants agissent donc en synergie, mais dans le cas de la pessière l'augmentation des apports acides due à l'effet filtrant des frondaisons est prépondérant, alors que dans la hêtraie, la forte immobilisation cationique dans la biomasse perenne joùe un plus grand rôle.

Mots clés : acidification, reboisement, bassin-versant, modélisation, MAGIC, granite, Mont-Lozère, hydrochimie.

\section{INTRODUCTION}

Les effets combinés des dépôts atmosphériques acides et des reboisements, qui entraînent la baisse de la productivité des écosystèmes et l'augmentation de l'acidité des eaux de surface ont été largement décrits pour les zones sensibles de l'Europe et de l'Amérique du Nord (MAYER et ULRICH, 1974 ; ROSENQUIST, 1980 ; KRUG et FRINK, 1983 ; MASON, 1990, PROBST et al., 1990). Des modèles ont été élaborés pour décrire les processus d'acidification et prédire l'évolution de la qualité des sols et des eaux sous différents scénarios de charges en polluants et d'utilisation des terres (CHRISTOPHERSEN et al., 1982 ; COSBY et al., 1985a et b ; GHERINI et al., 1985 ; WHITEHEAD et NEAL, 1987).

L'Europe du Sud-Ouest est également soumise à la pollution atmosphérique acide et au cours des 100 dernières années, d'importantes étendues ont été reboisées ou simplement abandonnées dans les zones marginales. Les problèmes d'acidification ne sont pas encore apparus dans ces régions car bien souvent les sols sont moins sensibles et des apports fréquents de poussières sahariennes riches en carbonate de calcium neutralisent en partie l'acidité des apports atmosphériques (LOYE-PILOT et al., 1986 ; RODA et al., 1990). Toutefois, la menace qui pèse sur les zones montagnardes aux sols minces et acides doit être évaluée, afin que des mesures puissent être prises rapidement. La réversibilité est en effet bien plus aisée lors des premiers stades de l'acidification (COSBY et al., 1985b; HAUHS et WRIGHT, 1988).

L'une des premières tentatives d'application du modèle MAGIC à des bassins-versants sud-européens est présentée ici. La version à un compartiment de ce modèle a été utilisée pour simuler les changements à long terme de la qualité des sols et des eaux dans trois petits bassins-versants granitiques du sud de la France, étudiés depuis 1981. 


\section{1 - TERRAIN D'ÉTUDE}

Les bassins versants du Mont-Lozère (Cloutasses : 81 ha ; Sapine : 54 ha ; Latte: $19,5 \mathrm{ha})$ sont situés au sud-est du Massif-Central, à $80 \mathrm{~km}$ de la mer, entre $1100 \mathrm{~m}$ et $1500 \mathrm{~m}$ d'altitude, sur un batholite granitique (fig. 1). Les types de sols représentés vont du ranker au sol brun ocreux et brun acide humifère. Le climat de la région est de type sub-méditerranéen montagnard.

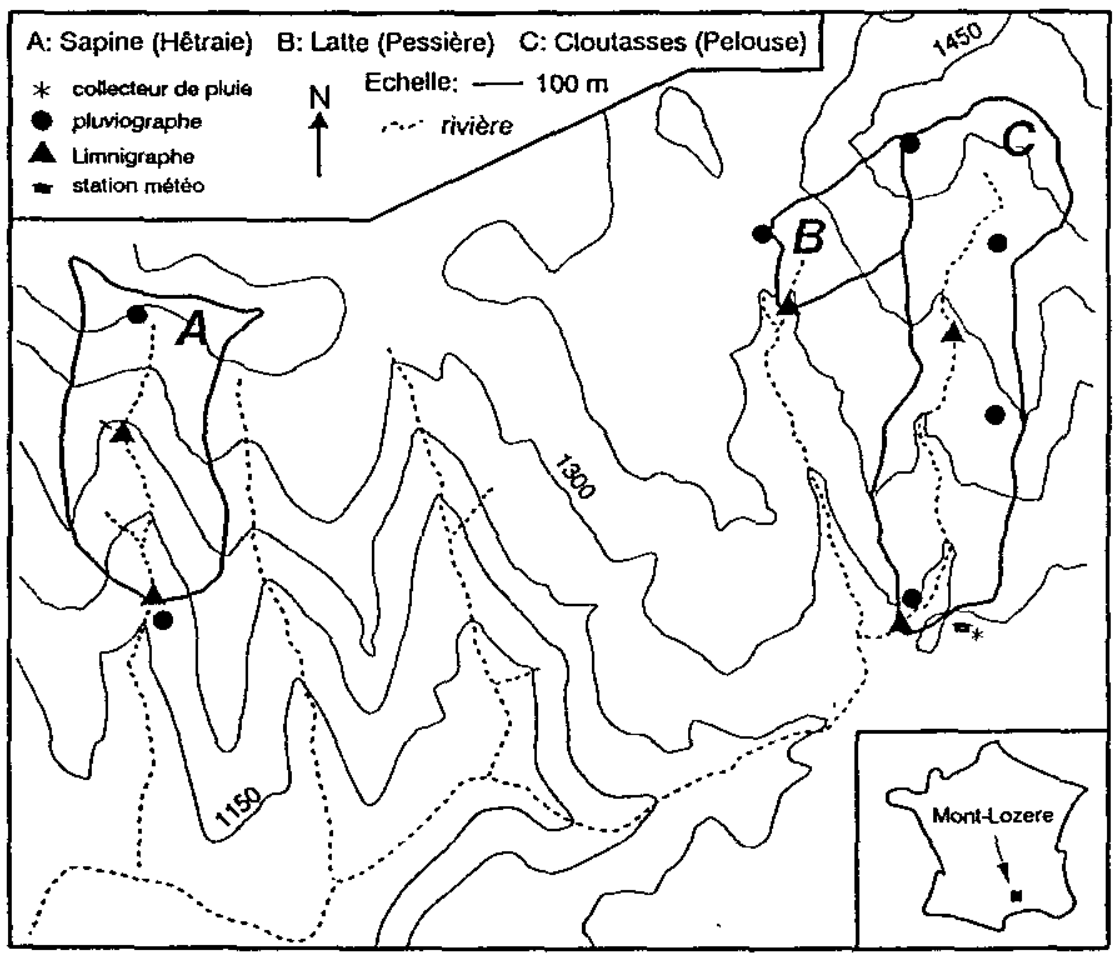

Figure 1 Localisation et équipement des bassins-versants du Mont-Lozère.

Mont-Lozère catchments : location and equipment.

Jusqu'au début de ce siècle, l'évolution du couvert végétal et de son exploitation anthropique a probablement été très semblable dans les trois bassins versants (PILLET, 1981). A la fin du Néolithique, le Mont-Lozère est presque entièrement couvert par une hêtraie-sapinière. La principale phase de défrichement a lieu au Moyen-Age, et le maximum de population et de pression pastorale se situe autour de 1850. Une progressive déprise agricole commence peu après, et là intervient la différenciation entre les bassins versants étudiés ici : des taillis de hêtres semi-naturels colonisent lentement les zones les moins propices au pâturage (bassin-versant de la Sapine); des plantations de résineux sont réalisées ailleurs (bassin de la Latte); un 
pâturage extensif d'ovins transhumants se maintient près des hameaux encore habités (bassin des Cloutasses).

Ces bassins versants font l'objet d'un suivi hydrologique (précipitations et débits à l'exutoire), et hydrochimique (prélèvement et analyse des eaux de pluie et de rivières) en continu depuis 1981. Les pluviolessivats ont aussi été prélevés dans les deux forêts pendant trois ans. Le $\mathrm{pH}$, la conductivité, l'aicalinité et les concentrations en $\mathrm{Ca}, \mathrm{Mg}, \mathrm{K}, \mathrm{Na}, \mathrm{Cl}, \mathrm{NH}_{4}, \mathrm{SO}_{4}, \mathrm{NO}_{3}$ et Si ont été déterminés par les méthodes classiques. Des descriptions détaillées de ces méthodes et des techniques de mesure sont données dans de précédentes publications (DUPRAZ, 1984 ; DURAND, 1989 ; LELONG et al., 1990).

\section{2 - LE MODÈLE MAGic}

Le modèle MAGIC (Model of Acidification of Groundwater in Catchments) est un modèle déterministe destiné à simuler les changements à long terme des propriétés chimiques des sols et des eaux dans des bassins versants soumis à des apports atmosphériques acides (COSBY et al., 1985a, 1990). Le schéma conceptuel du modèle est donné en figure 1 , et les principales équations qu'il utilise sont présentées au tableau 1. Le sol est considéré comme homogène sur l'ensemble du bassin et décrit par un jeu unique de paramètres représentant ses caractéristiques physico-chimiques moyennes : densité apparente (DA), porosité (PS), épaisseur (ES), capacité d'échange cationique $(C E C)$, taux de cations échangeables $\left(E_{c a}, E_{M g} \ldots\right)$. Une série de relations d'équilibre modélise les processus pédologiques et hydrochimiques majeurs, échange cationique (relations de Gaines et Thomas : équations 1 et 2), solubilité et mobilisation de Al (équation 3), adsorption de sulfate (isotherme de Langmuir : équation 4), altération chimique des minéraux $W$ (fournissant des cations à l'eau du sol), assimilation biologique $U$ (retirant des cations de l'eau du sol), dissociation des acides organiques de type $\mathrm{H}_{2} \mathrm{~A}$ (équation 5) et dissociation de lacide carbonique (due aux fortes pressions partielles de $\mathrm{CO}_{2}$ dans le sol: équations 6 à 8 ). Les entrées au système sont calculées en faisant le produit de la lame d'eau incidente (P) par les concentrations dans la pluie $\left((\mathrm{Ca})_{\mathrm{p}}\right)$ et par un facteur de dépôt sec (FD) dépendant de l'élément considéré et du couvert végétal (voir tableau 2 et partie 3). Une fois données les valeurs des différents paramètres el constantes thermodynamiques (tableaux 3 et 4), la composition chimique de l'eau du sol est la solution du système d'équations formé par ces relations. L'alcalinité est calculée par l'équation de la balance ionique. Pour calculer la composition chimique des eaux de rivières, le système est résolu une seconde fois à plus faible $\mathrm{pCO}_{2}$ (dégazage), en ignorant les relations d'échange cationique (l'eau n'étant plus en contact avec le sol). En d'autres termes, les composés non conservatifs lors du passage de la solution du sol vers cours d'eau sont le $\mathrm{pH}$, l'Aluminium, les acides organiques et l'acide carbonique: l'alcalinité et les concentrations en cations et anions majeurs restent constantes. L'équation de conservation de la masse (9) est ensuite appliquée à l'ensemble du système 
pour déterminer les variations des réserves d'éléments en présence, qui sont recalculées pour chaque année (10).

Tableau 1 Principales équations utilisées par le modèle MAGIC. La signification des symboles est donnée dans le texte. Les parenthèses représentent les concentrations molaires et les accolades, les activités chimiques. Les équations (1), (9) et (10) s'appliquent également à $\mathrm{Mg}$, ainsi qu'à $\mathrm{Na}$ et $\mathrm{K}$ en tenant compte de la valence.

Table 1 Main équations used by MAGIC (see texte). Brakets denote molar concentrations and braces activities. Equations (1), (9) and (10) also apply to $\mathrm{Mg}, \mathrm{K}$ and $\mathrm{Na}$.

$$
\begin{aligned}
& \frac{\left\{\mathrm{Ca}^{2+}\right\}^{3} \times \mathrm{E}_{\mathrm{Al}}^{2}}{\left\{\mathrm{Al^{3+ }}\right\}^{2} \times E_{\mathrm{Ca}}^{3}}=S_{\mathrm{Ca}} \\
& E_{A}+E_{C a}+E_{M g}+E_{N a}+E_{K}=1 \\
& \frac{\left.\{A\}^{3+}\right\}}{3}=K_{A I} \\
& \left\{\mathrm{H}^{+}\right\} \\
& \frac{E_{\mathrm{rx}} \times 2\left(\mathrm{SO}_{4}^{2-}\right)}{\mathrm{C}+2\left(\mathrm{SO}_{4}^{2-}\right)}=E_{S} \\
& \frac{[\mathrm{HA}] \times\left[\mathrm{H}^{+}\right]}{\left[\mathrm{H}_{2} \mathrm{~A}\right]}=\mathrm{K}_{1} \\
& \frac{\left(\mathrm{CO}_{2}(\mathrm{aq})\right\}}{\mathrm{P}_{\mathrm{CO}_{2}}}=\mathrm{K}_{\mathrm{CO}_{2}^{1}} \\
& \frac{\left[\mathrm{HCO}_{3}\right] \times\left\{\mathrm{H}^{+}\right\}}{\left\{\mathrm{CO}_{2}(\mathrm{aq})\right\}}=\mathrm{K}_{\mathrm{CO}_{2}^{2}} \\
& \frac{\left\{\mathrm{CO}_{3}^{2}\right\rceil \times\left\{\mathrm{H}^{+}\right\}}{\left(\mathrm{HCO}_{3}^{-}\right\}}=\mathrm{K}_{\mathrm{CO}_{2}^{3}} \\
& \mathrm{C} a_{T}=\mathrm{DA} \times \mathrm{ES} \times \mathrm{CEC} \times \mathrm{E}_{\mathrm{Ca}}+\mathrm{ES} \times \mathrm{PS} \times 2\left(\mathrm{Ca}^{2+}\right) \\
& \mathrm{dCa} / \mathrm{dt}=\mathrm{P} \times \mathrm{FD} \times 2\left(\mathrm{Ca}^{2+}\right)_{\mathrm{P}}+\mathrm{W}_{\mathrm{Ca}}-\mathrm{U}_{\mathrm{Ca}}-\mathrm{Q} \times 2\left(\mathrm{Ca}^{2+}\right)
\end{aligned}
$$

Dans cette application, le modèle a été utitisé pour reconstituer la composition chimique des rivières entre 1844 et 1984, sous différents scénarios de modification des taux d'assimilation biologique, de l'évaporation et du captage de dépôts secs et ce, en fonction de l'historique de l'évolution du couvert végétal exposée plus haut : les trois bassins étaient couverts de pelouse en 1844, puis une hêtraie et une pessière se sont développées sur deux des bassins. Les calibrations ont été réalisés en changeant les paramètres non mesurables jusqu'à ce que les valeurs simulées pour les sols et les rivières en 1984 coïncident avec les valeurs mesurées (moyennes pondérées de 1981 à 
1988, DURAND et $a l ., 1992 b)$. Ensuite, des simulations ont été réalisées pour les 150 années suivantes sous trois hypothèses d'évolution des émissions anthropiques de soufre.

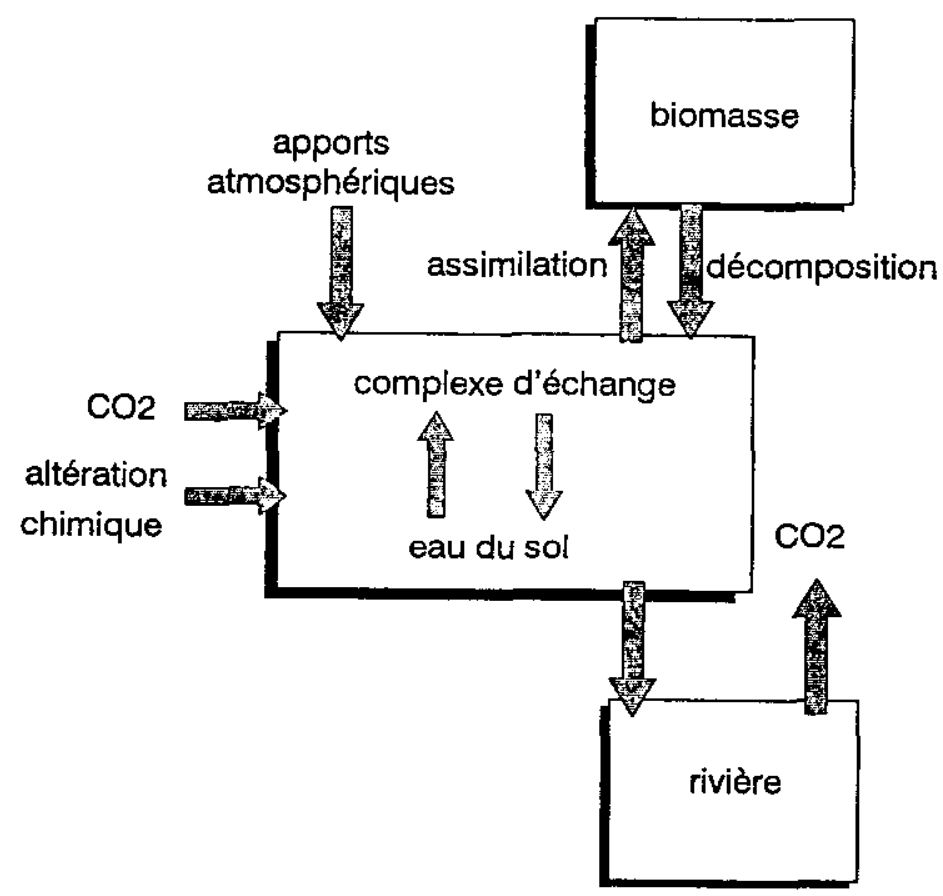

Figure 2 Schéma conceptuel du modèle MAGIC (d'après cosBY et al., 1990). Conceptuel diagram of the MAGIC model (after COSBY et al., 1990).

Tableau 2 Concentrations moyennes pondérées de l'eau de pluie et facteur de dépôt sec en forêt $\left(^{*}\right)$ : valeurs estimées. $\left(^{* *}\right)$ : moyenne 1981-1988.

Table 2 Volume weighted mean concentrations in rainwater and dry deposition factor in forest. (") : estimated values. (") : mean 1981-1988.

\begin{tabular}{|c|c|c|c|c|}
\hline \multirow[b]{2}{*}{ meq. $m^{-3}$} & \multicolumn{2}{|c|}{ Eau de pluie } & \multicolumn{2}{|c|}{ Facteur de dépôt sec } \\
\hline & $1844\left(^{\circ}\right)$ & $1984\left(^{* *}\right)$ & Hêtraie & pessière \\
\hline $\mathrm{Ca}$ & 25,0 & 32,5 & 1,2 & 1,4 \\
\hline $\mathrm{Mg}$ & 10,0 & 10,2 & 1,1 & 1,2 \\
\hline $\mathrm{Na}$ & 25,0 & 27,7 & 1,0 & 1,0 \\
\hline $\mathbf{K}$ & 2,0 & 4,1 & 1,2 & 1,4 \\
\hline NH4 & 0,0 & 20,6 & 1,0 & 1,0 \\
\hline $\mathrm{SO} 4$ & 15,0 & 65,7 & 1,3 & 1,6 \\
\hline$a$ & 29,4 & 29,4 & 1,0 & 1,0 \\
\hline NO3 & 5,0 & 20,7 & 1,0 & 1,0 \\
\hline $\mathrm{H}_{+}$ & 0,46 & 20,9 & & \\
\hline $\mathrm{pH}$ & 6,34 & 4,68 & & \\
\hline
\end{tabular}


Tableau 3 Caractéristiques physico-chimiques moyennes des sols. (") paramètres ajustés en calibrant le modèle.

Table 3 Lumped physico-chemical soil date. (*) calibrated parameters.

\begin{tabular}{|c|c|c|c|}
\hline & Cloutasses (pelouse) & Sapine (hêtre) & Latle (epicea) \\
\hline Epaisseur ES (m) & 0,70 & 0,70 & 0,70 \\
\hline Capacité d'échange CEC (meq.ko- ${ }^{-1}$ ) & 50 & 56 & 59 \\
\hline Capacité maximum d'adsorption de $\mathrm{SO}_{4}$ & 12,90 & 17,50 & 17,05 \\
\hline Emx (meq.kg-1) & $(*)$ & $(*)$ & $(*)$ \\
\hline $\begin{array}{l}\text { Concentration } \mathrm{SO}_{4} \text { a } 1 / 2 \text { saturation } \\
\text { d'adsorption }\end{array}$ & 45 & 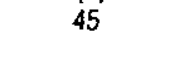 & 45 \\
\hline$C\left(m e q \cdot m^{-3}\right)$ & $(*)$ & (") & $(*)$ \\
\hline Porosité PS (\%) & 35 & 35 & 35 \\
\hline Densitế apparente $\left(\mathrm{kg} \cdot \mathrm{m}^{-3}\right)$ & 1400 & 1300 & 1400 \\
\hline Taux de saturation (\%) & 14,2 & 12,6 & 12,0 \\
\hline Ca échangeable $\mathrm{E}_{\mathrm{C}}(\%)$ & 7,3 & 5,9 & 6,0 \\
\hline Mg échangeable $\mathrm{E}_{M \mathfrak{v}}(\%)$ & 2,1 & 2,4 & 2,0 \\
\hline Kéchangeable $E_{K}(\%)$ & 1,2 & 1,0 & 1,0 \\
\hline $\mathrm{Na}$ échangeable $\mathrm{E}_{\mathrm{Na}}(\%)$ & 3,6 & 3,3 & 3.0 \\
\hline
\end{tabular}

Tableau 4 Autres paramètres du modèle (Valeurs fixées).

Table 4 Other model parameters (fixed values).

\begin{tabular}{|c|c|c|c|}
\hline & Cloutasses (pelouse) & Sapine (hêtre) & Latte (epicea) \\
\hline Lame d'eau précipitée $P$ (m) & 1,957 & 1,957 & 1,850 \\
\hline $\begin{array}{l}\text { Sols: } \\
\text { Coef. solubilité Al }\left(\log _{10}\right) \mathrm{pK} \mathrm{AL}_{\mathrm{AL}} \\
\mathrm{pCO}_{2} \text { (atm.) }\end{array}$ & & $\begin{array}{l}9,06 \\
0,316\end{array}$ & \\
\hline $\begin{array}{l}\text { Mat. org. dissoutes (mmol.m-3) } \\
\mathrm{pK}_{1} \text { acides organiques }\left(\log _{10}\right)\end{array}$ & 25 & $\begin{array}{l}35 \\
4,25\end{array}$ & 35 \\
\hline $\begin{array}{l}\text { Rivières: } \\
\text { Lame d'eau écoullée Q (m) } \\
\text { Mat org. dissoutes (mmol.m-3) } \\
\mathrm{pCO}_{2} \text { (atm.) }\end{array}$ & 1,550 & $\begin{array}{l}1,365 \\
0,0 \\
0,0032\end{array}$ & 1,250 \\
\hline Température moyenne $\left({ }^{\circ} \mathrm{C}\right)$ & 6,0 & 6,0 & 8,5 \\
\hline
\end{tabular}

\section{3 - SÉQUENCES DE DÉPOSITION, DE TAUX D'ASSIMILATION BIOLOGIQUE ET DE LAMES D'EAU ÉCOULÉES}

La séquence d'évolution des concentrations en $\mathrm{SO}_{4}$ dans la pluie utilisée pour la période 1844-1984 est basée sur l'évolution des émissions industrielles de soufre en Europe (fig. 3a). Le même schéma a été adopté pour les auters éléments, avec une amplitude de variation plus faible : les concentrations en sels marins et en cations basiques, qui dépendent principalement de la distance à la mer et des apports de poussières sahariennes, ont probablement peu variés (tableau 2). 
Le taux de captage des dépôts secs a été augmenté linéairement pendant la phase de fermeture de la végétation arborée. Les facteurs de dépôt actuels (FD) ont été estimés à partir des analyses de pluviolessivats (DURAND et al., 1992a). La croissance des deux forêts a eu lieu à une vitesse différente, par conséquent différentes durées ont été fixées pour cette phase dans les deux bassins : 1920-1970 pour la hêtraie, 1930-1945 pour la pessière (fig. 3b).

3a.
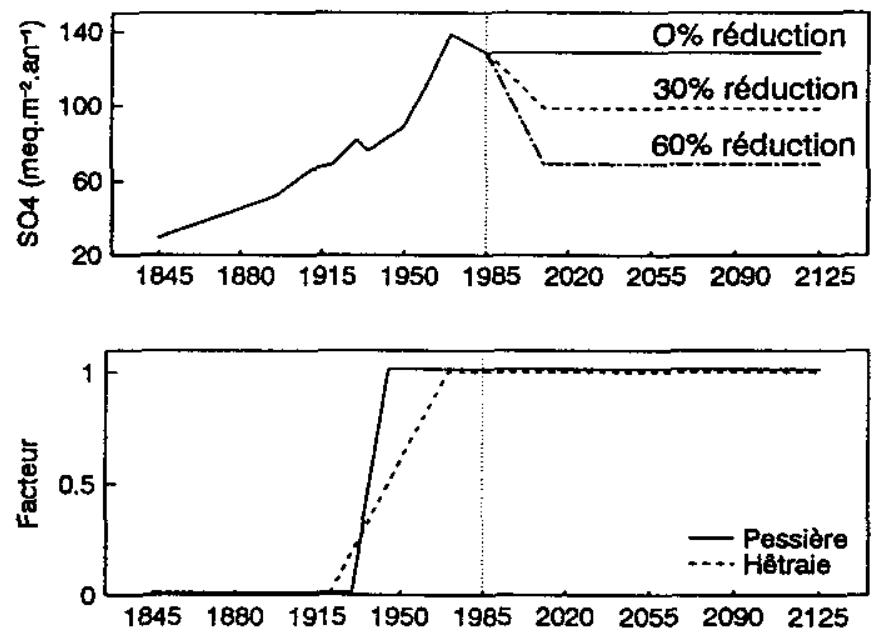

3c.

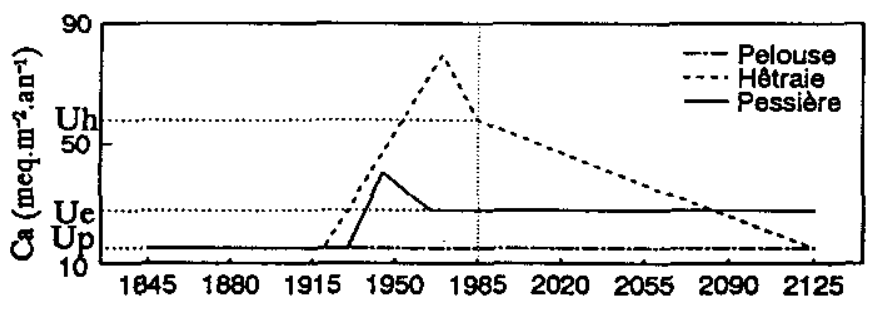

$3 d$.

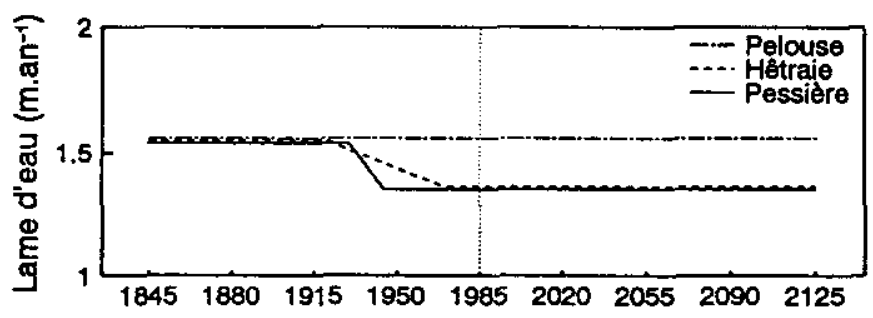

Figure 3 Séquences chronologiques utilisées pour les reconstitutions et les prévisions.

Sequences used for hindcasts and forecasts

2a : Apports de sulphate par les pluies ; $2 b$ : Dépôts secs : 1 = valeur des facteurs en $1984 ; 2 c$ : Prélèvements biologiques (exemple du calcium); $2 d$ : Lames d'eau écoulées annuelles. 2a: SO, wet deposition ; $2 \mathrm{~b}:$ Dry deposotion : $1=1984$ factors values ; $2 c$ : Biological uptake (example for $\mathrm{Ca}$ ) $; 2 \mathrm{~d}$ : Annual discharge. 
Il a aussi fallu tenir compte des variations des taux d'assimilation biologique. Dans le cas de la pelouse, ces taux d'assimilation ont été évalués d'après une étude bibliographique (DUPRAZ, 1984) et des mesures de la productivité fourragère et du pâturage (CABURET A., non publié). En dépit des grandes variations de la pression pastorale pendant la période considérée, une assimilation biologique constante a été supposée, afin d'illustrer les effets des reboisements sur une pelouse prise comme référence.

Pour la pessière, le taux d'assimilation biologique a été supposé atteindre un maximum 15 ans après la plantation, et décroître ensuite pendant 20 ans jusqu'à une valeur constante, conformément au schéma utilisé par COSBY et al. (1990) pour une pessière écossaise (fig. 3C). Dans la hêtraie, la dynamique de l'incorporation des nutriments est nécessairement différente car le peuplement n'est pas équien (fig. $3 C$ ). Pour les deux forêts, une première estimation basée sur les résultats de mesures de biomasse et de minéralomasse (HANSHI, non publié) a été ajustée dans la procédure de calibration (tableau 5). Les taux d'assimilation d'azote ont été considérés proportionnels aux apports atmosphériques, et choisis de façon à ajuster les teneurs en $\mathrm{NO}_{3}$ et $\mathrm{NH}_{4}$ des rivières.

Tableau 5 Taux d'assimilation biologique nette des trois types de végétation (voir fig. $2 b$ et texte). (*): ajusté en fonction du bilan hydrologique. $\left(^{* *}\right)$ : en pourcentage des entrées atmosphériques.

Table $5 \quad$ Net biological uptake for the three vegetation types (see fig. $2 b$ and texte). (*): adjusted according to the water balance. $\left(^{* *}\right)$ : percent of the atmospheric input.

\begin{tabular}{|cccc|}
\hline meq.m- - $^{-a^{-1}}$ & Peiouse (Up) & Hêtraie (Uh) & Pessière (Ue) \\
\hline $\mathrm{Ca}$ & 15,0 & 58,0 & 27,7 \\
$\mathrm{Mg}$ & 4,0 & 11,5 & 5,3 \\
$\mathrm{~K}$ & 2,0 & 4,5 & 2,7 \\
$\mathrm{Na}$ & 5,0 & 15,0 & 7,6 \\
$\mathrm{SO4}$ & 2,5 & 6,0 & 6,7 \\
$\mathrm{Cl}\left({ }^{*}\right)$ & 6,5 & 4,6 & 0,0 \\
$\mathrm{NH} 4 \%(* *)$ & 94,2 & 94,0 & 91,5 \\
$\mathrm{NO} \%\left(^{* *}\right)$ & 77,0 & 95,5 & 70,0 \\
\hline
\end{tabular}

Le déficit d'écoulement atteint $600 \mathrm{~mm}$ en forêt contre $400 \mathrm{~mm}$ en pelouse (LELONG et al., 1990). Par conséquent, une décroissance linéaire de la lame d'eau écoulée annuelle a été imposée pendant la croissance des forêts (fig. 3d).

Les hypothèses adoptées pour les simulations prospectives sont les suivants :

- pas de changements pour les prélèvements de nutriments par la pelouse (afin là encore de lui faire jouer le rôle de référence) et par la pes. sière. Pour cette dernière, il est bien évident qu'au minimum une coupe et une replantation devraient intervenir dans cette période. II a toutefois été considéré, en première approximation, que les effets en résultant se compensaient, ce 
qui équivaut à une assimilation biologique constante au niveau de celle d'une pessière d'âge moyen : cette hypothèse tend probablement à sous estimer l'impact acidifiant de ce type de sylviculture;

- décroissance linéaire des prélèvements par la hêtraie, en supposant un vieillissement naturel du peuplement, qui entraîne la diminution de la production annuelle de biomasse et l'augmentation du recyclage des nutriments.

Trois scénarios d'évolution des émissions anthropiques de soufre ont été testés : constantes au niveau de $1984,30 \%$ et $60 \%$ de réduction entre 1984 et 2010. Dans le dernier cas, les teneurs d'azote ont aussi été réduites de $60 \%$ et les teneurs des autres ions (cations et chlorures) de $10 \%$.

\section{4 - CAlibration}

La procédure de calibration a consisté principalement à ajuster les paramètres de l'adsorption de $\mathrm{SO}_{4}$, les taux d'altération chimique et la composition initiale du complexe d'échange de façon à faire coîncider les caractéristiques chimiques des sols et des rivières simulées et observées pour l'année 1984. Les paramètres du modèle rassemblés dans les tableaux 3 et 4 (sauf $E_{m x}, C$, et les taux de cations échangeables) ont été exclus de la calibration. Ce type de modèle permet, lorsqu'if s'applique valablement, un ajustement quasi « parfait " des variables cibles, c'est-à-dire les 10 paramètres chimiques des eaux de rivière (tableau 6) et les quatre paramètres du complexe adsorbant des sois (tableau 3). Cette propriété vient de la grande sensibilité du modèle à un nombre important de paramètres optimisés. Il en résulte que les valeurs de ces paramètres ajustés n'ont que peu de signification en soit: elles ne doivent être interprétées que relativement aux autres applications du modèle. Cette sensibilité ne concerne toutefois que la reconstitution des valeurs pour une année précise : elle ne s'applique pas à l'allure des évolutions à l'ong terme produit par le modèle. Celle-ci dépend en effet essentiellement des séquences chronologiques d'apports atmosphériques et de taux d'assimilation biologique, ainsi que de l'ensemble des données mesurées pour l'année de référence (une analyse détaillée de la sensibilité et des performances du modèle est exposée par HORNBERGER et al., 1986).

\subsection{Adsorption de $\mathrm{SO}_{4}$ dans les sols}

Les valeurs optimisées des deux paramètres de l'isotherme de Langmuir, $E_{m x}$ (capacité d'adsorption maximale) et $C$ (concentration dans la solution de sol à la demi-saturation) sont très proches dans les deux forêts $\left(E_{m x} \approx\right.$ 17 meq. $\mathrm{kg}^{-1}$ et $C=45$ meq. $\mathrm{m}^{-3}$ ) ; dans la pelouse, $C$ est identique et $E_{m x}$ un peu plus faible (12,9 meq. $\mathrm{kg}^{-1}$, tableau 3 ). Ces valeurs sont cohérentes avec les résultats d'analyses de sols qui indiquent des teneurs en soufre total de l'ordre de 50 meq. $\mathrm{kg}^{-1}$ dans thorizon A des sols forestiers (LELONG et al., 1989). 
Tableau 6 Concentrations moyennes pondérées annuelles des rivières, simulées (1984) et observées (moyennes 1981-88, sauf pour Latte: 1981-1986).

Table 6 Annual flow-weighted mean concentration in streamwater: simulated (1984) and observed (1981-88 mean, except Latte: 1981-1986).

\begin{tabular}{|crrrrrr|}
\hline & \multicolumn{2}{c}{ Cloutasses } & \multicolumn{2}{c}{ Sapine } & \multicolumn{2}{c|}{ Latte } \\
meq. ms & sim. & obs. & sim. & obs. & sim. & obs. \\
\hline $\mathrm{Ca}$ & 55,8 & 55,8 & 41,3 & 41,2 & 67,6 & 67,8 \\
$\mathrm{Mg}$ & 25,1 & 25,1 & 25,4 & 25,4 & 42,7 & 42,8 \\
$\mathrm{Na}$ & $\mathbf{4 7 , 7}$ & 47,7 & 51,8 & 51,8 & 54,1 & 54,1 \\
$\mathrm{~K}$ & 8,1 & 8,1 & 6,0 & 5,9 & 7,6 & 7,5 \\
$\mathrm{NH}_{4}$ & 1,5 & 1,5 & 1,8 & 1,8 & 2,5 & 2,0 \\
$\mathrm{SO}_{4}$ & 1,5 & 1,5 & 1,8 & 1,8 & 2,5 & 2,0 \\
$\mathrm{Cl}$ & 32,9 & 32,9 & 39,8 & 39,8 & 45,2 & 45,1 \\
$\mathrm{NO}_{3}$ & 6,0 & 6,0 & 1,4 & 1,3 & 10,3 & 10,0 \\
$\mathrm{pH}$ & 5,8 & 5,8 & 5,8 & 5,8 & 5,8 & 5,8 \\
$\mathrm{Alc}$. & 40,8 & 36,0 & 20,0 & 17,9 & 35,2 & 35,0 \\
\hline
\end{tabular}

Ces niveaux traduisent une forte capacité de rétention du soufre. Dans la quasi-totalité des applications précédentes de MAGIC, les valeurs d'E $m x$

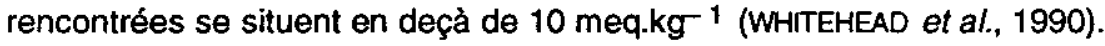

\subsection{Taux d'altération chimique et conditions initiales}

Les taux d'altération en $\mathrm{K}$ et $\mathrm{Na}$ sont similaires dans les trois bassins versants (tableau 7). Le bassin de la hêtraie présente un faible taux d'altération en $\mathrm{Ca}$, et le bassin de la pessière affiche un très fort taux d'altération en $\mathrm{Mg}$ comparé aux deux autres bassins. Si les paramètres du modèle ont une signification physique, cela implique que la composition chimique de la rochemère diffère sensiblement d'un bassin à l'autre.

Il en résulte que les conditions initiales sont légèrement différentes d'un bassin à l'autre : l'alcalinité et les concentrations en cations sont plus faibles dans la hêtraie, alors que le taux de saturation en bases est plus bas dans la pessière.

Tableau 7 Taux d'altération et coefficients de sélectivité optimisés.

Table 7 Otptimised weathering rates and selectivity coefficients.

\begin{tabular}{|c|c|c|c|}
\hline & Cloutasses (pelouse) & Sapine (hêtre) & Latte (epicea) \\
\hline \multicolumn{4}{|c|}{ Taux d'altération (meq. $m^{-2} \cdot a^{-1}$ ): } \\
\hline Calcium $\left(W_{c a}\right)$ & 29,0 & 20,5 & 26,2 \\
\hline Magnésium $\left(W_{M a}\right)$ & 17,9 & 14,3 & 32,0 \\
\hline Sodium $\left(W_{\mathrm{N} 2}\right)$ & 19,7 & 16,0 & 14,4 \\
\hline Potassium $\left(W_{K}\right)$ & 9,5 & 9,0 & 8,5 \\
\hline \multicolumn{4}{|l|}{ Coefficients de sélectivité : } \\
\hline Calcium $\left(\log _{10}\right)\left(\mathrm{S}_{\mathrm{Ca}}\right)$ & 0,32 & $-0,31$ & 0,55 \\
\hline Magnésium $\left(\log _{10}\right)\left(S_{M a}\right)$ & 0,92 & 0,23 & 1,40 \\
\hline Sodium $\left(\log _{10}\right)\left(S_{\mathrm{N}_{2}}\right)$ & $-1,94$ & $-1,89$ & $-1,67$ \\
\hline Potassium $\left(\log _{10}\right)\left(\mathrm{S}_{\mathrm{k}}\right)$ & $-5,65$ & $-6,19$ & $-5,65$ \\
\hline
\end{tabular}




\section{5 - ÉVOLUtion À LONG TERME de LA CHIMIE des SOLS ET DES RIVIÈRES}

Les résultats des simulations présentent de notables différences d'un bassin à l'autre (fig. 4 et 5). La réponse du bassin non forestier à l'augmentation de lacidité incidente est très modérée. Même dans l'hypothèse «pessimiste " (pas de réductions des émissions anthropiques de soufre pendant les 150 prochaines années), la qualité des sols et des eaux reste relativement inchangée : le $\mathrm{pH}$ ne descend pas au-dessous de 5,5, l'alcalinité de la rivière reste positive $\left(11,5\right.$ meq. $\mathrm{m}^{-3}$ ) et les réserves initiales de cations échangeables sont diminuées de $30 \%$ seulement.

Dans la pessière, une chute de $\mathrm{pH}$ et d'alcalinité est décelée à partir des années 70 , quand les apports de soufre et l'assimilation biologique étaient à leur maximum. La désaturation cationique des sols s'amorce seulement dix ans plus tard, car jusqu'alors les dépôts supplémentaires de cations sur le feuiliage compensaient les pertes. Dans les cas où il n'y a pas de réduction des apports de soufre ou dans le cas d'une réduction de $30 \%$ d'ici à 2010 , l'acidification du ruisseau continue jusqu'à la fin de la simulation, avec une ampleur qui implique une détérioration des réserves de cations basiques échangeables (diminués de moitié par rapport à 1844) et surtout de la qualité des eaux ( $\mathrm{pH}$ de 4,6, alcalinité de -33 meq. $\mathrm{m}^{-3}$ ). Dans le cas d'une réduction de $60 \%$ des apports de soufre, seule une lente amélioration est décelable, mais les dommages restent limités.

Dans la hêtraie, c'est limpact de la végétation, plutôt que les dépôts acides, qui domine l'évolution du système : même dans le cas d'une absence de réduction des apports, une amélioration est observée quand les prélèvements biologiques diminuent suite au vieillissement du peuplement. A ce stade (qui intervient entre 2030 et 2100 , suivant les scénarios), les apports supplémentaires de cations dus aux dépôts secs permettent la restauration des réserves de cations échangeables. La réduction de ces réserves a débuté dès 1920, quand la forêt s'est installée. Pourtant, la chute de $\mathrm{pH}$ et d'alcalinité n'intervient qu'après 1970, comme dans le cas de la pessière. Dans le scénario pessimiste, l'appauvrissement chimique du sol et la détérioration de la qualité des eaux entre 1970 et 2100 est aussi sévère qu'à la Latte, à la fois à cause de la saturation progressive de la capacité d'adsorption de soufre et des forts taux d'assimilation biologique.

Ces différences entre les deux forêts ne sont pas seulement dues aux différences de végétation. Les paramètres du modèle contrôlant la proportion de cations adsorbés (coefficients de sélectivité) sont très différents dans les deux bassins (tableau 7). Par exemple, pour une réserve initiale totale donnée de $\mathrm{Ca}$, la concentration aqueuse dans le sol est bien plus forte (environ 1,6 fois) dans le cas de la pessière que dans le cas de la hêtraie, où cet élément sera plus fortement adsorbé. En conséquence, dans le bassin de la hêtraie, les variations dans les bilans entrées-sorties se traduisent par des phénomènes d'adsorption/désorption avant que la composition des eaux à l'exutoire ne change vraiment. Dans le bassin couvert d'épicéas au contraire, les 
mêmes changements affecteront d'abord les concentrations en rivière: l'inertie chimique du sol étant plus grande, la réversibilité des processus d'acidification en cas de réduction des apports sera plus lente.
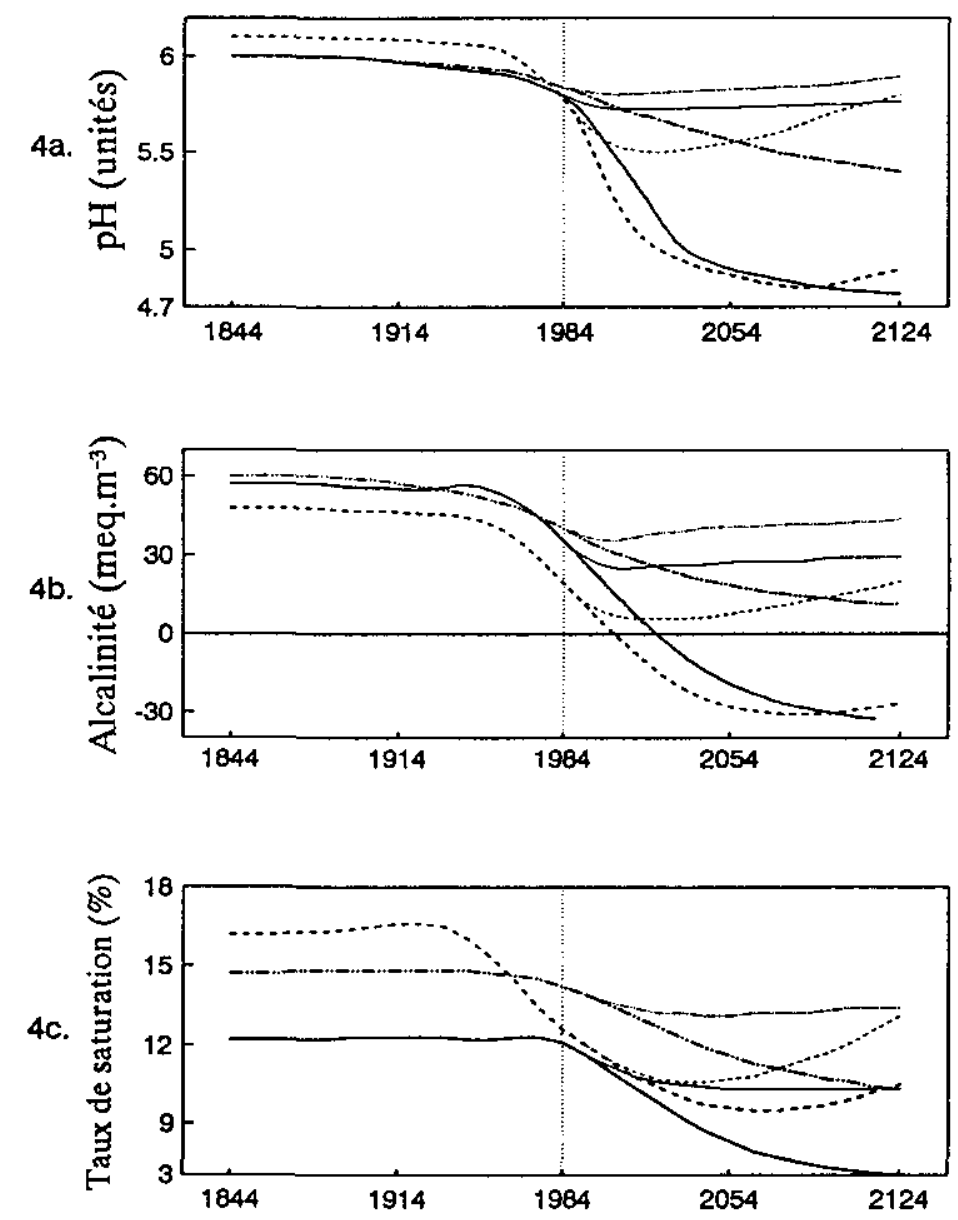

Figure 4 Evolutions simulées pour les trois bassins versants sous deux scénarios de pollution: pas de réduction des apports de soufre (traits épais) et $60 \%$ de réduction entre 1984 et 2010 (traits fins). (-) : Latte (épicéas) ; $(--)$ : Sapine (hêtres) ; (- - - ) : Cloutasse (pelouse).

Simulated changes for the three catchments under two air pollution scenarios : no reduction of sulfur deposition (thick lines) and $60 \%$ reduction between 1984 and 2010 (fine lines). (-): Latte (spruce) ; (- -): Sapine (beech) ; $(-\cdots-)$ : Cloutasse (grassland). 

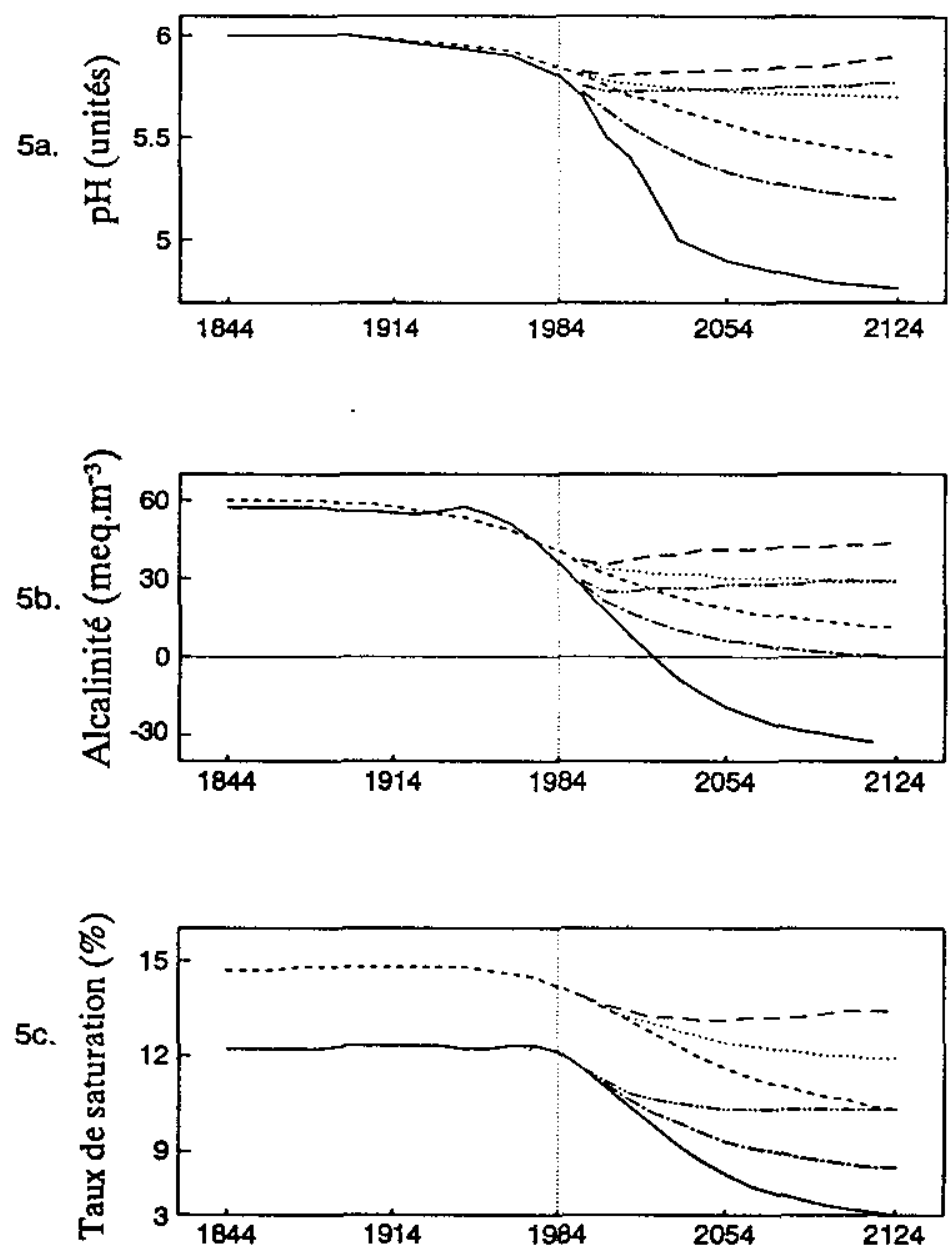

Figure 5 Evolutions simulées pour la Latte (épicéas) et les Cloutasses (pelouse) en fonction des trois scénarios de dépôts de soufre:

- pas de réduction: $(-)$ : épicéas, (--) : pelouse.

$-30 \%$ de réduction : $(-\cdots)$ : épicéas, $(\cdots)$ : pelouse.

$-60 \%$ de réduction: $(-\cdots-)$ : épicéas, $(--)$ : pelouse.

Simulated changes for the la Latte (spruce) and Les Cloutasses (grassland) catchments under three scénarios of sulfur deposition:

- no reduction: (-) : spruce, (- - ) : grassland.

$-30 \%$ reduction: $(-\cdots)$ : spruce, ( . .) : grassland.

$-60 \%$ reduction: (- - - ) : spruce, $(--)$ : grassland. 


\section{CONCLUSION}

Cette application du modèle MAGIC confirme le rôle important des variations des taux d'assimilation biologique dans les processus d'acidification. Leur évaluation est difficile et imprécise (COSBY et al., 1990), mais pourtant indispensable pour une simulation pertinente des impacts des reboisements et des déboisements.

Le modèle a pu reproduire le fonctionnement actuel des trois écosystèmes étudiés et fournit des informations très précieuses sur leur évolution future selon différents scénarios. Les simulations montrent que le reboisement et la pollution atmosphérique peuvent agir en synergie dans l'acidification des bassins versants. Ceci corrobore les conclusions d'études du même type sur des bassins versants britanniques (NEAL et al., 1986, JENKINS et al., 1990). Cependant, dans ce contexte géographique particulier, le couvert forestier peut aussi aider à renverser les effets de l'acidification dans le cas où la réduction des apports atmosphériques va de pair avec une réduction de l'assimilation de nutriments par les arbres aux dépens du sol, ce qui intervient dans le cas de cycles forestiers de longue durée. Par contre, dans le cas de rotations courtes de type plantation de résineux, cette étude montre que la dégradation à court terme de la qualité des eaux et des sols est une menace qui n'épargne pas les régions méridionales.

\section{REMERCIEMENTS}

Ce travail a été réalisé grâce à une bourse sectorielle de la CCE (BSTEP/900019). Les auteurs sont reconnaissants à A. JENKINS et P.G. WHITEHEAD pour leurs critiques, à A. HANSCHI pour la communication de données inédites, et à J.F. DIDON-LESCOT et G. DROUET pour le travail de terrain et de laboratoire.

\section{RÉFÉRENCES BIBLIOGRAPHIQUES}

CHRISTOPHERSEN N., SEIP H.M., WRIGHT R.F., 1982. A model for streamwater chemistry at Birkenes, Norway. Water Resour. Res., 18 (4) : 977-996.

COSBY B.J., WRIGHT R.F., HORNBERGER G.M., GALLOWAY J.N., 1985a. Modelling the effects of acid deposition : estimation of long-term water quality responses in a small forested catchment. Water Resour. Res., 21 : 1591-1601.

COSBY B.J., HORNBERGER, G.M., GALLOWAY J.N., WRIGHT R.F., 1985b. Time scales of catchment acidification. Environ. Sci. Technol., 19(12) : 1144-1149. 
COSBY B.J., JENKINS A., FERRIER R.C., MILLER J.D., WALKER T.A.B., 1990. Modelling stream acidification in afforested catchments : long-term reconstructions at two sites in central Scotland. J. Hydrol., $120: 143-162$.

DUPRAZ C., 1984. Bilans des tansferts d'eau et d'éléments minéraux dans trois bassins versants comparatifs à végétation contrastée. Thèse Doc. Ing., Univ. d'Orléans, $363 p$.

DURAND P., 1989. Biogéochimie comparée de trois écosystèmes (pelouse, hêtraie, pessière) de moyenne montagne granitique (Mont-Lozere, France). Thèse Univ. d'Orléans, $186 \mathrm{p}$.

DURAND P., LELONG F., NEAL C., 1992a. Comparison and significance of annual hy drochemical budgets in three small granitic catchments with contrasting vegetation (Mont-Lozere, France). Accepté pour publication dans Environmental Pollution.

DURAND P., NEAL C. et LELONG F., DIDONLESCOT J.F., 1992b. Hydrochemical variations in spruce, beech and grassland areas, Mont-Lozere, Southern France. Accepté pour publication dans J. Hydrol.

GHERINI S.A., MOK L., HUDSON R.J.M., DAVIS G.F., CHEN C.W., GOLDSTEIN R.A., 1985. The ILWAS model : formulation and application. Water, Air, Soil Pollut., 26(4) : 425-460.

HAUHS M., WRIGHT R.F., 1988. Acid deposition : reversibility of soil and water acidifica. tion - a review -. CCE, Air Pollution Report $n^{\circ} 11.42 \mathrm{p}$.

HORNBERGER G.M., COSBY B.J., GALLO WAY J.N., 1986. Modeling the effects of acid deposition : uncertainty and spatial variability in estimation of long-term sulfate dynamics in a region. Water Resour. Res., 22(8) : 1 293-1 302.

JENKINS A., COSBY B.J., FERRIER R.C., WALKER T.A.B. et MILLER J.D., 1990. Modelling stream acidification in afforested catchments : an assessment of the relative effects of acid deposition and afforestation. J. Hydrol, 120 : 163-181.

KRUG E.C., FRINK C.R., 1983. Acid rain on acid soil : a new perspective. Science, 221 : 520-525.

LELONG F., DURAND P., VANNIER C., GUILLET B., ROUILLER J., 1989. Importance de la rétention des ions sulfates dans des sols granitiques acides en région de moyenne montage (Mont-Lozère). Relation générale avec le pouvoir tampon des sols. C.R. Acad. Sci., Paris, 309(2) : 19911996.

LELONG $F$., DUPRAZ C., DURAND P., DIDON-LESCOT J.F., 1990. Effects of vegetation type on the biogeochemistry of small catchments (Mont-Lozere, France). J. Hydrol. 116 : 125-145.

LOYE-PILOT M.D., MARTIN J.M., MORELLI J., 1986. Influence of Saharan dust on the rain acidity and atmospheric input to the Mediterranean. Nature, 321 : 427-428.

MASON B.J., 1990. The Surface Water Acidification Programme. Cambridge Univ. Press, Cambridge, U.K., 522 p.

MAYER R., ULRICH B., 1974. Conclusions on the filtering action of forests from ecosystern analysis. Oecol. Plant., 9(2) : 157-168.

NEAL C., WHITEHEAD P.G., NEALE R., COSBY B.J., 1986. Modelling the effects of acidic deposition and conifer afforestation on stream acidity in the British Uplands. J. Hydrol, 86 : 15-26.

PILLET P., 1981. Recherche sur l'organisation et l'évolution des unités écologiques du Parc National des Cévennes. Rapport final P.N.C., Florac, $110 \mathrm{p}$.

PROBST A., DAMBRINE E., VIVILLE D., FRITZ B., 1990. Influence of acid atmospheric inputs on surface water chemistry and mineral fluxes in a declining spruce stand within a small granitic catchment (Vosges Massit, France). J. Hydrol., 116 : 101124.

RODA F., AVILA A., BONILLA D., 1990. Precipitation, through-fall, soil solution and strearnwater chemistry in a holm-oak (Quercus ilex) forest. J. Hydrol., 116 : 345-363.

ROSENQUIST I.T., 1980. Influence of forest vegetation and agriculture on the acidity of fresh water. In : advances in Environmental Science and Engineering, Vol. 3. Gordon and Breach, 56-79.

WHITEHEAD P.G., NEAL C., 1987. Modelling the effect of acid deposition in upland Scotland. Transactions of the Royal Soc. of Edimburg, 78 : 385-392.

WHITEHEAD P.G., JENKINS A., COSBY B.J., 1990. Modelling long-term trends in surface water acidification. In: The Surface Waters acidification programme, B.J. Mason, Cambridge Univ. Press, 431-443. 